\title{
Ultrafast exciton dynamics in semiconductors: Effects of disorder and confinement
}

\author{
J.M. Hvam, V.G. Lyssenko, and D. Birkedal \\ J. Erland \\ Fysisk Institut, Odense Universitet, DK-5230 Odense M, Denmark
}

Mikroelektronik Centret, The Technical University of Denmark, Bldg. 345 east, DK-2800 Lyngby, Denmark.

\begin{abstract}
The dynamics of excitonic transitions in semiconductors have been investigated by degenerate four-wave mixing experiments. We have studied the coherence, interference and dephasing of free, bound and localized excitons in bulk semiconductors and of quasi-two-dimensional excitons in quantum well structures. The influence of inhomogeneous broadening is investigated and compared with quantum interference in a continuum of states. The nature of four-wave mixing beats in a system of bound excitons and biexcitons is discussed.
\end{abstract}

\section{INTRODUCTION}

Coherent and incoherent exciton dynamics, on a femtosecond timescale, is efficiently studied by degenerate four-wave mixing (DFWM) and transient grating experiments with ultrashort laser pulses. ${ }^{1}$ Transient coherent DFWM experiments, involving free polarization decays or photon echoes, can supply useful information about the initial scattering mechanisms after an optical excitation, ${ }^{2.3 .4}$ and in some cases quantum beats between such coherently prepared states can give additional knowledge about oscillator strengths, energy splittings and shifts. ${ }^{3,6,7}$ Experimental distinction between such quantum beats and classical polarization interferences between indenpendent transitions has recently caught attention because of the possibility of determining the degree of interaction between different excited states in a semiconductor. ${ }^{8.9}$

We have previously measured, and reported, the dephasing times of free, bound and localized excitons in various semiconductors as a function of temperature and exciton density, and thereby identified the principal scattering mechanisms and rates. 'In the present work we shall concentrate on some of the more subtle aspects of the ultrafast dynamics and interferences in exciton systems, including quasi-two-dimensional excitons in quantum well structures. Before we discuss these results, we shall describe in the next sections the principles of the applied spectroscopies, that are transient degenerate fourwave mixing and nonlinear quantum beats.

\section{TRANSIENT FOUR-WAVE MIXING}

In general, four-wave mixing is the mixing of three input waves in a nonlinear medium, setting up a third-order nonlinear polarization, which in turn serves as an antenna for a fourth outgoing signal wave. In standard nonlinear optics terms, the thirdorder nonlinear polarization is expressed as

$$
P^{(3)}(k, \omega)=\chi^{(3)}\left(\omega ; \omega_{1}, \omega_{2}, \omega_{3}\right) \cdot E_{1}\left(k_{1}, \omega_{1}\right) E_{2}\left(k_{2}, \omega_{2}\right) E_{3}\left(k_{3}, \omega_{3}\right)
$$

where $\chi^{(3)}$ is the third order nonlinear susceptibility, and the frequency and the wavevector of the collimated signal beam are given by the energy and wavevector conservations, expressing the phase-matching conditions

$$
\omega= \pm \omega_{1} \pm \omega_{2} \pm \omega_{3} \wedge k= \pm k_{1} \pm k_{2} \pm k_{3}
$$

where $+/-$ is entered for absorbed/emitted waves. 
With two degenerate beams $\left(\omega_{1}=\omega_{2}=\omega\right.$ and $\left.k_{1}=k_{2}=k\right)$, impinging under an angle $\theta\left(\cos \theta=k_{1} \cdot k_{2} / k^{2}<1\right)$, as in Fig. 1, there are two possibilities with perfect phase-match: $k=k_{1}$ and $k=k_{2}$. These nonlinear signals are generated in the directions of the incident beams and may therefore be difficult to detect at moderate intensities. In thin samples, there are also two possibilities with near phase-match: $\mathbf{k}=2 \mathbf{k}_{2}-\mathbf{k}_{1}-\Delta \mathbf{k}$ and $\mathbf{k}^{\prime}=2 \mathbf{k}_{1}-\mathbf{k}_{2}-\Delta \mathbf{k}$, where $\Delta \mathbf{k}$ is the wavevector mismatch perpendicular to the sample plane. This geometry has the advantage that the nonlinear signal is a collimated beam propagating in a background free direction. It is therefore well suited for the detection of even very small nonlinear signals. The linear background can to a high degree be eliminated by simple spatial filtering. Performing DFWM with ultrashort laser pulses, one can obtain not only spectral information about the nonlinear coefficients, but also dynamical information about the optical excitations. The type of information obtained depends on the actual experimental configuration, as well as on the character of the samples investigated. Transient DFWM is a modification of the excite-and-probe technique, where the pump consists of two beams, split off the same coherent laser beam, setting up a coherent polarization grating in the medium, and the probe is either one of the pump beams, self-diffracting in this grating (two-beam configuration, Fig. 2a) or a third delayed beam diffracting in the grating set up by the two first pulses (three-beam configuration, Fig. $2 b$ ). In the following, we shall examine the two-beam configuration where the two incident laser pulses are split off the same laser pulse, and are impinging on the sample with a variable optical delay between them.

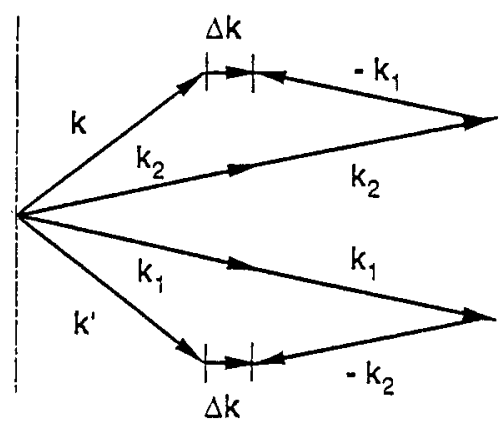

Fig.1 Wavevector conservation for DFWM with two light beams, $\mathbf{k}_{1}$ and $\mathbf{k}_{2}$

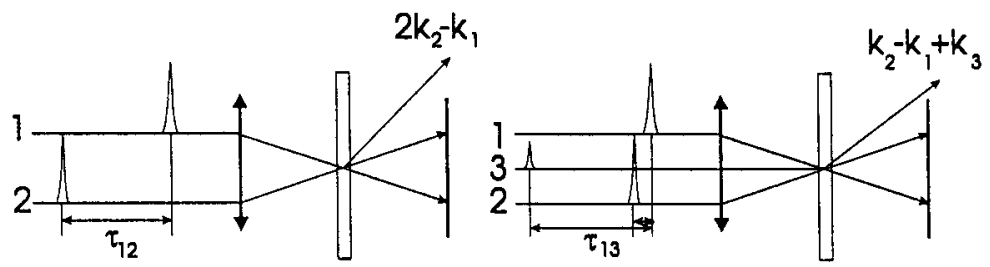

b)

Fig. 2. Transient DFWM with two beams (a) and three beams (b), respectively.

In order for the two laser pulses to interact coherently in the nonlinear medium, for example by setting up a polarization grating, the delay between them should not exceed the dephasing time of the nonlinear polarization caused by the first laser pulse. The nonlinear DFWM signal is then self-diffraction of the second pulse in the grating set up by the coherent overlap between the polarizations from the first and the second pulse. For pulse $\# 1$ arriving first $(\tau>0)$ as in Fig. 2a, a signal will thus be emitted in the direction $2 k_{2}-k_{1}$ as indicated. This result is obtained by solving the two-level optical Bloch equations to third order, which for $\delta$-pulses yields 10,11

$$
P^{(3)}(r, t, \tau>0) \propto \theta(t) \mu_{10}^{4} e^{i\left[\left(2 k_{2}-k_{1}\right) \cdot r-Q_{10}+a_{10}^{*} \tau\right]}
$$

where $t$ is the time after the arrival of the second pulse, $\Theta(t)$ is the Heaviside step function, and $\Omega_{10}=\omega_{10}$ - $i \gamma_{10}$ is the complex transition frequency with the damping $\gamma_{10}=1 / \mathrm{T}_{2}+1 /\left(2 \mathrm{~T}_{1}\right) . \mathrm{T}_{1}$ and $\mathrm{T}_{2}$ are the longitudinal and transverse relaxation times, respectively. The intensity of the DFWM signal in the direction $2 \mathbf{k}_{2}-\mathbf{k}_{1}$ is then

$$
I_{D F H M} \propto\left|P^{(3)}(t, \tau>0)\right|^{2} \propto \theta(t) \mu_{10}^{8} e^{-2 \gamma_{10}(t+\tau)}
$$

The corresponding signal in the frequency domain, as experimentally obtained by a spectrometer with a slow detector, and mathematically by Fourier transforming Eq. (3), is

$$
I_{D F W M} \propto\left|P^{(3)}(\omega, \tau>0)\right|^{2} \propto \frac{\mu_{10}^{8}}{\left|\Omega_{10}-\omega\right|^{2}} e^{-2 Y_{10} \tau}
$$

where $\omega$ is the optical frequency of the detected signal. Thus, spectra of the time-integrated DFWM signal contain information about the dephasing rate $\gamma_{10}$ as well as the resonance enhancements of the nonlinear signal.

If the above two-level system is inhomogeneously broadened, similar expressions can be obtained by integrating over 
the inhomogenous distribution of transition frequencies, e.g. $g\left(\omega_{10}\right) \propto \exp \left(-2 \ln 2\left(\omega_{10^{-}} \omega_{0}\right)^{2} / \Gamma_{10}{ }^{2}\right\}$, where $\omega_{0}$ is the center frequency and $\Gamma_{10}$ is the inhomogeneous intensity linewidth (FWHM).

$$
P_{i n h}^{(3)}(t, \tau) \propto \int_{0}^{\infty} P_{i n h}^{(3)}(t, \tau) g\left(\omega_{10}\right) d \omega_{10}
$$

In this case, destructive and constructive interferences between the different transition frequencies play a significant role, and the signal in the $2 \mathbf{k}_{\mathbf{2}}-\mathbf{k}_{\mathbf{1}}$ direction appears as a photon echo (PE) at the time $\mathrm{t}=\tau$.

$$
I_{P E} \propto\left|P_{i n h}^{(3)}(t, \tau>0)\right|^{2} \propto \mu_{10}^{8} e^{-\frac{\Gamma_{10}^{2}(t-\tau)^{2}}{16 \ln 2}} e^{-2 \gamma_{10}(t * \tau)}
$$

and the spectrally resolved time-integrated PE takes, for large delays $\left(\tau \geq 4 / \Gamma_{10}\right)$, the form

$$
I_{P E} \propto\left|P_{i n h}^{(3)}(\omega, \tau>0)\right|^{2} \propto \mu_{10}^{8} e^{-8 \ln 2 \frac{\left(\omega_{0}-\omega\right)^{2}}{\Gamma_{10}^{2}}} e^{-4 \varphi_{10} \tau}
$$

which for the same homogeneous damping decays twice as fast as the free polarization decay in Eq. (5). From a comparison of the observed DFWM decay with the linewidth, as observed in absorption or emission, it can be decided whether the transition is homogeneously or inhomogeneously broadened. Hence, the dephasing time, and thereby the homogeneous linewidth, can be determined from either Eq. (5) or Eq. (8).

\section{NONLINEAR QUANTUM BEAT SPECTROSCOPY}

The DFWM decays, or correlation traces, of Eqs. (5) and (8) assumed the sytems to be two-level like. If, on the other hand, there is a multiplicity of nearly degenerate excited levels, the decay of the nonlinear signal will be affected much the same way as for quantum beats in resonance fluorescence experiments. However, the quantum interferences, or beats, will in this case also appear in the correlation traces. The first pulse arrives at the sample at $t=-\tau$ and sets up a (linear) polarization that will decay according to the dephasing time, and show interference modulations in the case of nearly degenerate coherently excited states. The free polarization decay is responsible for the fluorescence decay as well as for the nonlinear interaction with the second pulse arriving at $t=0$. It is intuitively obvious that the integrated intensity of the nonlinear signal will carry over the beat modulation into the correlation trace of the nonlinear signal, as can be calculated by solving, to third order, the Bloch equations for a three-level system. ${ }^{12}$

Assuming the complex frequencies of the three-level system $\Omega_{j 0}=\omega_{j 0}-\gamma_{j 0}(j=1,2)$ and $\Omega_{21}=\omega_{21}=\omega_{20}-\omega_{10}$, the Bloch equations can be solved to third order using a Greens functions technique, to obtain an expression for the third order nonlinear polarization, propagating in the $2 \mathbf{k}_{\mathbf{2}}-\mathbf{k}_{\mathbf{1}}$ direction

$$
\begin{aligned}
& P^{(3)}(t, \tau>0) \propto \theta(t)\left[2 \mu_{10}^{4} e^{-t\left(O_{10} t-Q_{10}^{*} \tau\right)}+2 \mu_{20}^{4} e^{-t\left(\Omega_{20}-a_{20}^{*} \tau\right)}\right. \\
& \left.+\mu_{10}^{2} \mu_{20}^{2}\left(e^{-i\left(Q_{10 t}-Q_{20}^{*}\right)}+e^{-i\left(Q_{20}-Q_{10}^{*} \tau\right)}\right)\right]
\end{aligned}
$$

where $t$ is the time elapsed after the arrival of the second pulse. The intensity of the DFWM signal is proportional to the absolute square of the nonlinear polarization. For equal dipole matrix elements of the two transitions, the oscillatory behaviour of the signal as a function of real time $t$, as well as a function of the delay time $\tau$, is shown in Fig. 3a. Notice that also as a function of $t-\tau$, well defined oscillations can be observed. ${ }^{8}$

In an experiment where real-time resolution is not achieved, the maximum information is obtained by spectrally resolving the time-integrated nonlinear signal. In this case, the measured polarization is the Fourier transform of Eq. (9)

$$
\tilde{P}^{(3)}(\omega, \tau>0) \propto\left[\frac{2 \mu_{10}^{4} e^{i Q_{10}^{*} \tau}+\mu_{10}^{2} \mu_{20}^{2} e^{1 Q_{20}^{*} \tau}}{\Omega_{10}-\omega}+\frac{2 \mu_{20}^{4} e^{i Q_{20}^{*} \tau}+\mu_{10}^{2} \mu_{20}^{2} e^{i Q_{10}^{*} \tau}}{\Omega_{20}-\omega}\right]
$$


The two terms illustrate the resonance enhancement of the signal near each of the involved resonances, and the quantum beats appear in each of the terms. Detecting the signal near the first resonance, the second term can be neglected, and in this case the nonlinear signal can be expressed ${ }^{9}$

$$
I_{F W M} \propto \frac{4 \mu_{10}^{8} e^{-2 \gamma_{10} \tau}+\mu_{10}^{4} \mu_{20}^{4} e^{-\gamma_{20} \tau}+4 \mu_{10}^{6} \mu_{20}^{2} e^{-\left(\gamma_{10}+\gamma_{20} \tau\right.} \cos \left(\omega_{21} \tau\right)}{\left(\omega_{10}-\omega\right)^{2}+\gamma_{10}^{2}}
$$

revealing the modulation. Assuming again equal dipole matrix elements of the transitions and distinctely different dampings of the two levels involved in the beating, e.g. $\gamma_{20} \gg \gamma_{10}$, a simpler expression is obtained

$$
I_{F F M} \propto \frac{e^{-2 \gamma_{10} 0^{*}}\left[1+e^{-\gamma_{20} \tau} \cos \left(\omega_{21} \tau\right)\right]}{\left(\omega_{10}-\omega\right)^{2}+\gamma_{10}^{2}}
$$

showing that the average signal decays with the slowest damping rate, whereas the decay of the beat modulation decays with the fastest damping rate. Both damping rates can thus be determined from such a nonlinear quantum beat experiment. In the above is assumed a homogeneously broadened system with a free polarization decay, but a similar modulation of the correlation trace is obtained with inhomogeneous broadening, ${ }^{13}$ i.e. in a photon echo experiment, as first shown by Lampert, Compaan and Abella. ${ }^{14}$ If, however, the observed modulations are due to classical polarization interferences occuring in the detector, then they will be smeared out by inhomogeneous broadening.

In a homogeneously broadened system it may be difficult to distinguish between true quantum beats, e.g. from a threelevel system, and polarization interferences from independen two-level systems. For two independent two-level systems with the complex transition frequencies $\Omega_{j 0}=\omega_{j 0}-\mathrm{i} \gamma_{j 0}$, the solution is straight forward. For $\tau 0$ (no signal for $\tau<0$ ), the total third order polarization has the form

$$
P^{(3)}(t, \tau>0) \propto \theta(t)\left[\mu_{10}^{4} N_{1} e^{-l\left(Q_{10} t-Q_{10}^{*} \tau\right)}+\mu_{20}^{4} N_{2} e^{-l\left(Q_{20} t-Q_{20}^{*} \tau\right.}\right]
$$

where $t$ is the time after the arrival of pulse \#2, and $N_{j}$ is a complicated function of the time-integrated pulse amplitude, its spectral overlap with the resonance number $j$ and the concentration of two-level centers of this type. The FWM signal intensity is proportional to the absolute square of this polarization, and the temporal interference results from the cross term which has a phase that varies as $t-\tau$, as seen in Fig. $3 b$, in agreement with the experiments by Koch et al.. ${ }^{8}$

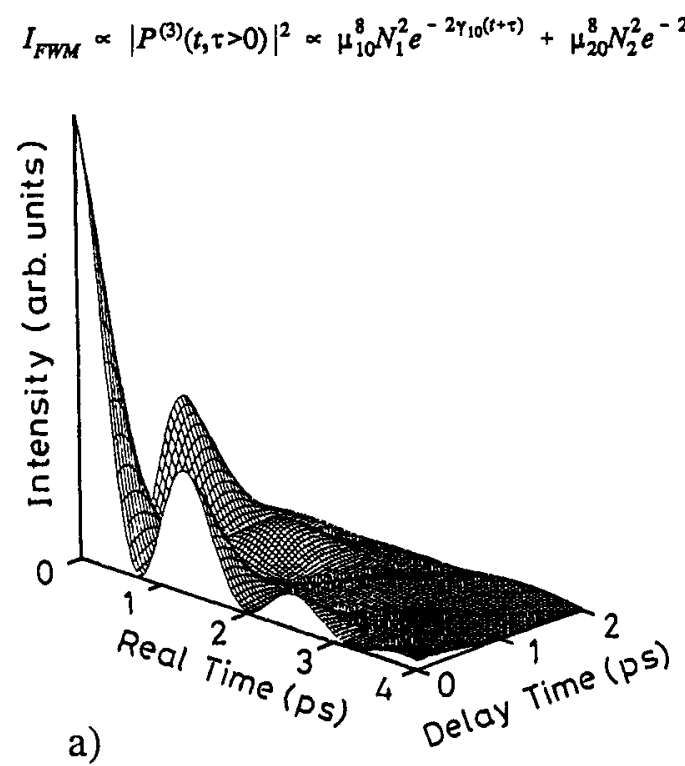

Fig. 3. Calculated FWM-signal for quantum beats (a) and for polarization interference (b). 
The spectral dependence is again obtained as the Fourier transform of Eq. (13)

$$
\tilde{P}^{(3)}(\omega, \tau>0) \times\left[\frac{N_{1} M_{10}^{4} e^{i 0_{10}^{* \tau}}}{\Omega_{10}-\omega}+\frac{N_{1} M_{20}^{4} e^{i 0_{20}^{* \tau}}}{\Omega_{20}-\omega}\right]
$$

and the intensity of the signal is proportional to the absolute square of Eq. (15). Now the modulation is due to the interference from the cross term, that contains both resonance denominators. Thus, if we detect the signal near the resonance $\omega_{10}$ the following expression is valid ${ }^{9}$

$$
I_{F T M} \propto \frac{N_{1}^{2} e^{-2 \gamma_{10} \tau}}{\left(\omega_{10}-\omega\right)^{2}+\gamma_{10}^{2}}+\frac{2 N_{1} N_{2} e^{-\gamma_{20} \tau} \cos \left(\omega_{21} \tau\right)}{\left(\omega_{10}-\omega\right)\left(\omega_{20}-\omega\right)+\gamma_{10} \gamma_{20}}
$$

The important thing to notice here is that the modulation of the nonlinear signal, as a function of $\tau$, will undergo a phase shift of $\pi$, when passing through either one of the resonances. This is in contrast to the true quantum beat in Eq. (12) and therefore offers an experimental possibility of distinguishing the two phenomena, as we shall see.

\section{COHERENT EXCITON DYNAMICS}

In this section, we shall discuss some selected aspects of coherent exciton dynamics in semiconductors. We take examples from quasi-two-dimensional excitons in GaAs/AlGaAs quantum wells, where we study exciton dynamics under the influence of confinement and disorder (interface roughness), ${ }^{15,16}$ and from bound excitons in CdSe, where we study the coupling and interferences between various bound complexes. ${ }^{17}$

\subsection{GaAs/AlGaAs quantum wells}

Figure 4 shows an example of the coherent exciton dynamics as revealed by a two-beam transient DFWM experiment in a specially prepared MBE-grown sample with a series of GaAs multiple quantum well (MQW) structures (10 periods) with well widths $\mathrm{L}_{\mathrm{z}}=80 \AA, 100 \AA, 130 \AA$, and $160 \AA$ and with $\mathrm{Al}_{0.3} \mathrm{Ga}_{0.7}$ As barriers of width $150 \AA$. From the DFWM spectra recorded as a function of the delay between the input pulses, a number of observations are made.

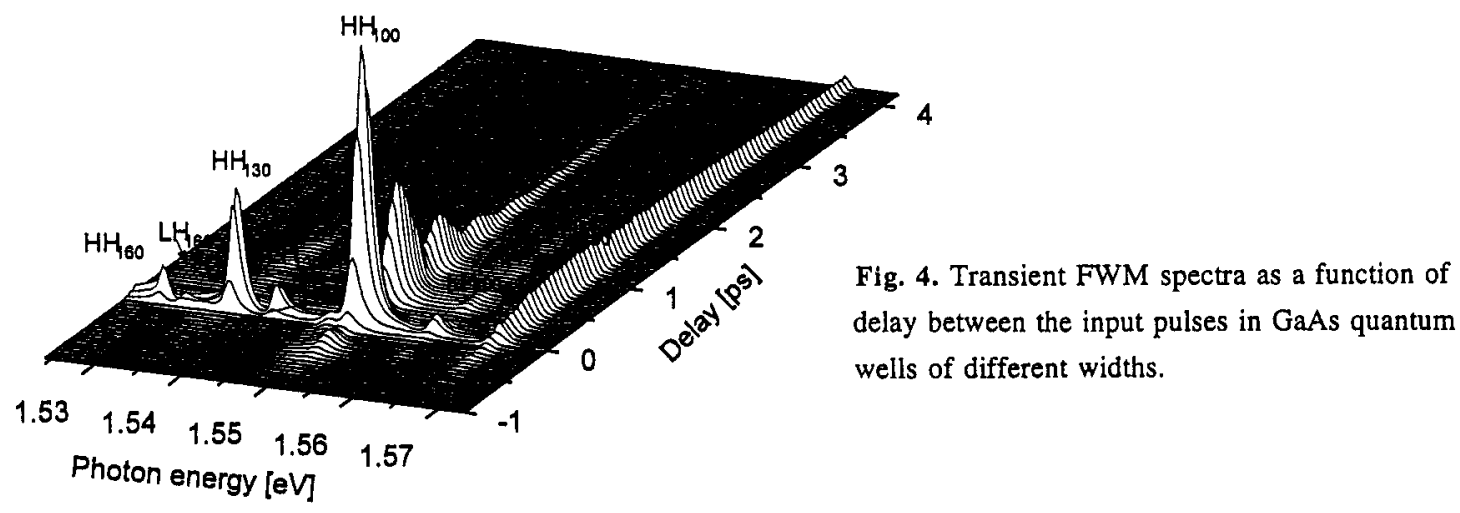

The strong resonance enhancement of the nonlinear signal (see Eqs. (5) and (8)) around the fundamental excitonic resonances, heavy hole excitons $(\mathrm{HH})$ and light hole excitons $(\mathrm{LH})$, is clearly observed for the different MQW series. The asymmetric behaviour of the DFWM signal as a function of the delay is in agreement with the simple two-level models in Eqs. (5) and (8) and should thus reveal the dephasing time $T_{2}=1 / \gamma_{10}$ of the corresponding transitions. However, the large differences observed in the decays has to be interpreted with care. In Fig. 4, the laser line is centered around the $\mathrm{HH}_{100}$ line, but is sufficiently broad to excite all the resonances observed. The DFWM signals from the wider well resonances decay much faster than the signals from the narrower wells, suggesting shorter dephasing times for the wider wells. One reason could be that the wider wells are excited well above the resonances, so that higher excited exciton states and continuum states contribute to the dephasing of the HH and LH excitons. A corresponding broadening of the resonances is, however, not observed in Fig. 4, so instead we conclude that the fast decay of the signal is due to destructive interference between the $\mathrm{n}=1 \mathrm{HH}(\mathrm{LH})$ excitons and the higher excited exciton states and continuum states. ${ }^{18} \mathrm{~A}$ wave packet is formed as a coherent superposition of many excited states, and the corresponding polarization dies out in a time that is inversely proportional to the spread in energy of excited states, which is essentially the laser line width, i.e the polarization decays almost as fast as the laser pulse. 
In Fig. 4, only the $\mathrm{HH}_{80}$ excitons are excited without a significant contribution from $\mathrm{LH}$ excitons and higher excited states. Therefore the observed decay is representative of the dephasing of $\mathrm{HH}$ excitons in GaAs MQW's at low density and low temperature. The observed dephasing time is about $10 \mathrm{ps}$, and the signal maximum for a finite positive delay indicates that the transition is inhomogeneously broadened and the signal appears as a photon echo., ${ }^{4.8}$ Note the difference between the interferences in an inhomogeneous distribution of independent oscillators and the destructive inteferences in a strongly coupled multi-level (continuum) system. In the former case, rephasing can take place and a signal can be detected for large delays (photon echo, Eq. (8)). In the latter case no rephasing occurs, and a signal is only observed for very small delays. ${ }^{18}$

The pronounced modulation observed on the $\mathrm{HH}_{100}$ line is due to a quantum beat between the $\mathrm{HH}_{100}$ and the $\mathrm{LH}_{100}$ resonances. The $\mathrm{HH}$ excitons and the LH excitons within the same MQW series are created in a coherent superposition state by the spectrally broad laser pulse, and the resulting polarization oscillates in time with a frequency $\omega_{21}=\Delta \mathrm{E} / \hbar$, where $\Delta E$ is the splitting of the $\mathrm{HH}$ and $\mathrm{LH}$ excitons (see Eq. (12)). As a result, the DFWM signal oscillates with the period $2 \pi / \omega$ as a function of delay. Note that no phase shift of the modulation is observed when going through the resonances (HH and $\mathrm{LH}$ ), confirming that it is a true quantum beat as in a three-level system (see Eqs. (11) and (12)). Similarly, beats between HH excitons and biexcitons have been observed, confirming the formation of biexcitons in the GaAs quantum wells. ${ }^{15.19} \mathrm{~A}$ signal for negative delays is a signature of polarization interactions ${ }^{20,21}$ and is clearly seen for the narrower wells. In particular for the $100 \AA$ wells, is seen a line (XX) that is dominant for negative delays. This line is due to the biexciton state associated with the heavy hole exciton resonance $\mathrm{HH}_{100}$ of the $100 \AA$ quantum wells, as can be ascertained by polarization experiments. The shift of the $\mathrm{XX}$ line from the $\mathrm{HH}$ line is equal to the biexciton binding energy, as also revealed by the period of the corresponding quantum beat. ${ }^{15.19}$

The observed dephasing times are slightly larger than in bulk GaAs. One difference is scattering due to interface roughness. If this is on a length scale smaller than the exciton radius, electrons and holes are scattered individually giving faster dephasing. If the interface roughness is on a length scale larger than the exciton radius (island formation) there will be a tendency of localization of the excitons which may lead to slower dephasing. In some samples, island formation is observed in DFWM by a splitting of the exciton resonances, corresponding to one monolayer well width fluctuations. The splitting is more clearly observed in the nonlinear signal than in the corresponding linear resonances of the sample. Figure 5 shows an example of the DFWM spectra in a $100 \AA$ quantum well sample as a function of the delay between the interfering. pulses.

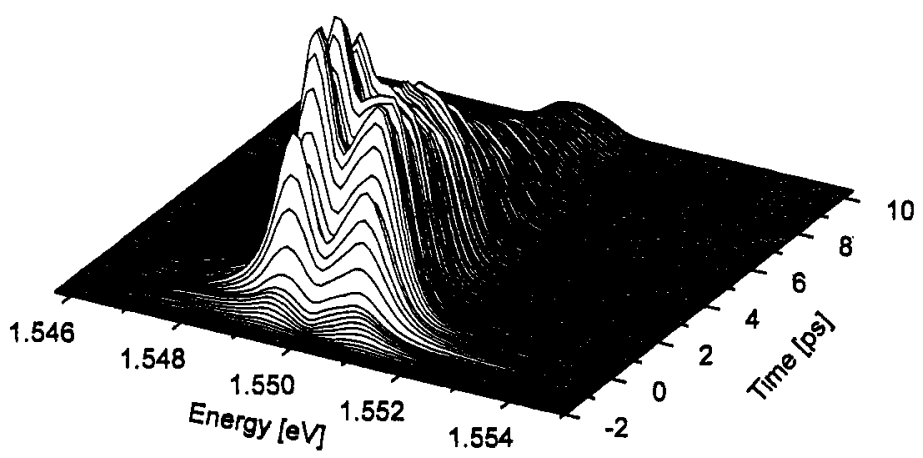

Fig. 5. Exciton splitting due to interface roughness as a function of delay.

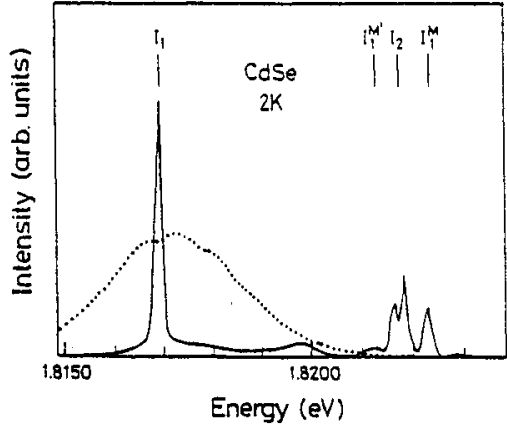

Fig. 6. DFWM spectrum for zero delay (solid curve) excited by laser pulse (dashed spectrum)

It is characteristic that the observed splitting is pronounced for small delays and disappears for larger delays, where only one (average) resonance is observed. We propose that this behaviour is due to nonlocality of the excitonic wavepackets resulting in a strong coupling of the excitons in the different spatial regions of the quantum wells. Quantum beats between excitons in different well width regions have previously been reported. ${ }^{22}$ It should be emphasized that this is an interference effect in the coherent exciton wave packets and not related to spatial diffusion of excitons between the regions of different well widths. An exciton density grating, set up by two interfering pulses with zero delay, shows the excitons in the different spatial regions distinctly separated spectrally for their entire lifetime. This is in agreement with previous reports of distinct luminescence lines from such island formation..$^{23}$

\subsection{Bound excitons in $\mathrm{CdSe}$}

In Fig. 4, polarization interferences between signals from different quantum wells were not observed. We have tested the distinction between true quantum beats and polarization interferences between independent two-level systems in the bound exciton systems observed in bulk CdSe at low temperature. ${ }^{1,717}$ We coherently excited several bound exciton states and 


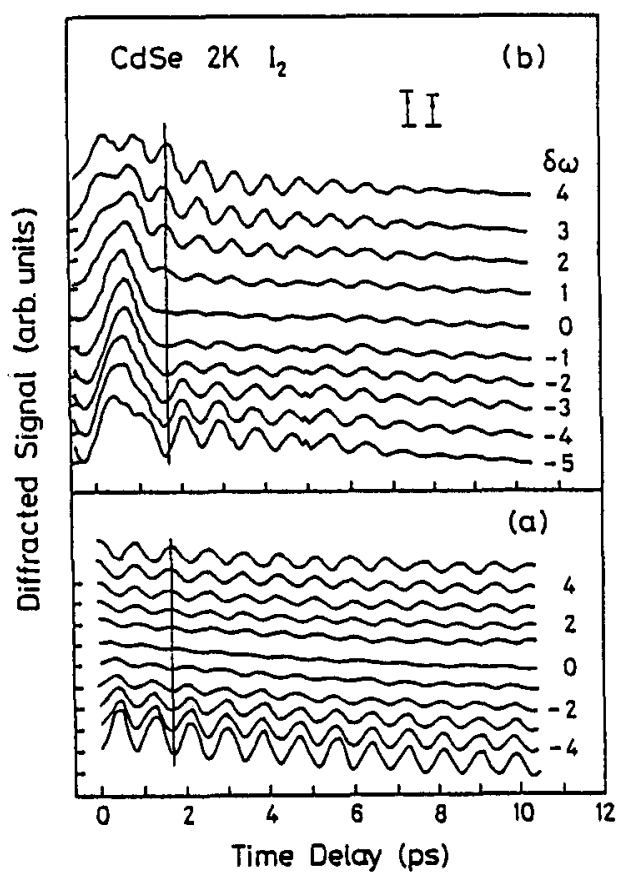

Fig.7. DFWM signal for different detunings (in steps of $0.08 \mathrm{meV}$ ) around the $\mathrm{I}_{2}$-line, (a) calculated from Eq. (16), and (b) experimentally.

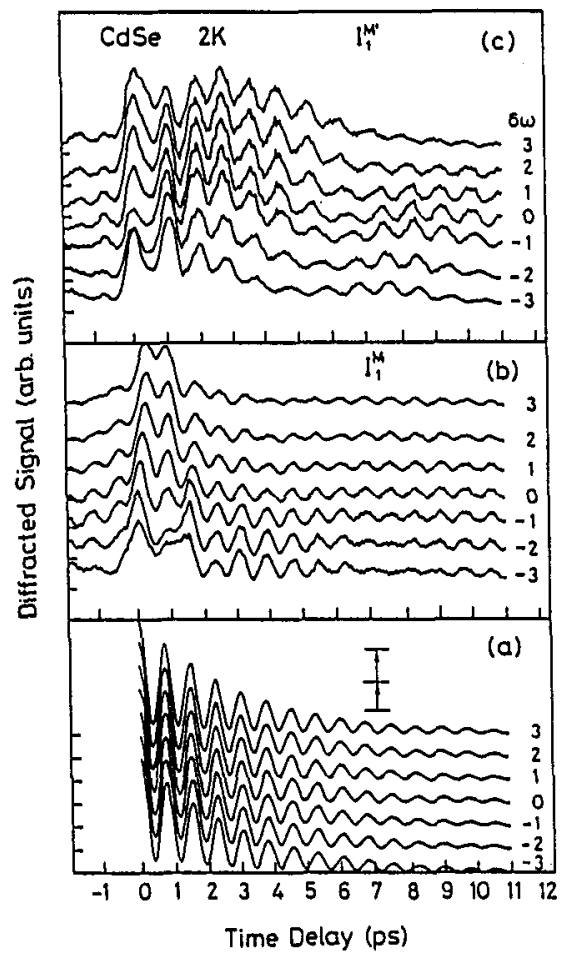

Fig. 8.DFWM signal for different detunings (in steps of $0.06 \mathrm{meV}$ ) around the $\mathrm{I}_{1}^{\mathrm{M}}$ and $\mathrm{I}_{1}{ }^{\mathrm{M}}$ lines, (a) calculated from Eq. (11), and (b) experimentally.

observed the beats in the resonantly enhanced four-wave mixing signal. For excitation near the $\mathrm{I}_{1}$-line (exciton bound to neutral acceptor) the four-wave mixing signal is strongly enhanced at the positons of the $I_{1}$-line, the $I_{2}$-line (exciton bound to neutral donor) and the $I_{1}{ }^{M}$ and $I_{1}{ }^{M}$ lines (biexciton bound to neutral acceptor), as shown in Fig. 6.

All the observed lines show strong modulation of the signal as a function of the delay between the interfering pulses and the results demonstrate that spectral resolution of the nonlinear signal makes it possible to distinguish between quantum beats and polarization interference. ${ }^{9}$ Figure 7 shows spectrally resolved correlation traces at the spectral positon of the $I_{2}$-resonance, as described in Sec. 3. At the $I_{2}$-resonance, $\hbar \omega=1.8218 \mathrm{eV}$, a phase shift of about $\pi$ and a minimum of the amplitude of the modulation is observed. This is analogous to the calculation from Eq. (16), shown in the lower part of Fig. 7. Hence, the $I_{1}-I_{2}$ beats are due to polarization interference, and excitons bound to different impurities can be considered as non-interacting.

The correlation traces of the self-diffracted signal, detected in the spectral region of the $I_{1}{ }^{M}$ and the and $I_{1}{ }^{{ }^{\prime}}$ bands are shown in Fig. 8. In contrast to the $I_{1}-I_{2}$ bound exciton polarization interference in Fig. 7 , no phase shift was observed near the resonance frequency $\omega_{0}\left(I_{1}{ }^{M}\right.$ and $\left.I_{1}{ }^{M}\right)$, proving that the involved transitions are within the same quantum system as calculated from Eq. (12) and shown in the lower part of Fig. 8. The spectrally broad laser pulse prepares a coherent mixture of neutral acceptor bound excitons $\left(A^{0} X\right)$ and acceptor bound biexciton complexes $\left(A^{0} M\right)$ by two-photon transitions, either directly or via the $A^{0} X$ resonance. The beating FWM processes are the transitions from the ground state $\left(A^{0}\right)$ to $A^{0} X$ and from $A^{0} X$ to $A^{0} M$. Since the $A^{0} M$ complex contains three holes, one hole must be in an excited state (2P, 3D). The $A^{0} X$ center is electonically equivalent to a neutral donor $D^{0}$, which explains why the $A^{0} \mathrm{M}$ center is energetically close to the $I_{2}$-line ( $D^{0} X$ ), which also is known to have excited hole states.

\section{CONCLUSIONS}

Ultrafast nonlinear spectroscopy has proven to be an extremely powerful tool to investigate the exciton dynamics in semiconductors on the time scale of the fastest scattering mechanisms in the semiconductor. Scattering rates can be determined from the coherent experiments and a number of different interference phenomena demonstrate new possibilities of retreiving information, hidden under inhomogenous broadening in linear spectroscopy. These interference phenomena provide a new tool to investigate the degree of interaction, or coupling, between states involved in the observed four-wave mixing beats. 


\section{ACKNOWLEDGEMENTS}

The authors are grateful to I. Balslev and K.-H. Pantke for valuable discussions and to C.B. Sørensen for growing the GaAs/AlGaAs quantum well samples. The work was supported by the Danish Natural Science Research Council.

\section{REFERENCES}

1. J.M. Hvam, J. Erland, and K.-H. Pantke, "Ultrafast exciton dynamics in direct gap semiconductors," in Ultrafast Phenomena in Semiconductors, Edited by D.K. Ferry and H.M van Driel, SPIE Proc. Vol. 2142 (1994) pp. 39-57.

2. A.M. Weiner, S. Silvestri, and E.P. Ippen, "Three-pulse scattering for femtosecond dephasing studies: theory and experiments," J. Opt. Soc. Am. B 2, 654 (1985).

3. L. Schultheis, J. Kuhl, A. Honold, and C.W. Tu, "Ultrafast phase relaxation of excitons via exciton-exciton and exciton-electron collisions," Phys. Rev. Lett. 57, 1635 (1986).

4. C. Dörnfeld and J.M. Hvam, "Optical nonlinearities and phase coherence in CdSe studied by transient four-wave mixing," IEEE J. Quantum Electron. QE-25, 904 (1989).

5. V. Langer, H. Stolz, and W. von der Osten, "Observation of quantum beats in the fluorescence of free excitons," Phys. Rev. Lett. 64, 854 (1990).

6. E.O. Göbel, K. Leo, T.C. Damen, and J. Shah, "Quantum beats of excitons in quantum wells," Phys. Rev. Lett. 64, 1801 (1990).

7. K.-H. Pantke, B.S. Razbirin, V.G. Lyssenko, J. Erland, and J.M. Hvam, "Nonlinear quantum beat spectroscopy in CdSe," Proc. 21st. Int. Conf. Phys. Semiconductors, Beijing 1992, edited by P. Jiang and H.-Z. Zeng (World Scientific Publishing Co., Singapore, 1992) pp. 129-136.

8. M. Koch, J. Feldmann, G. von Plessen, E.O. Göbel, and P. Thomas, "Quantum beats versus polarization interference: an experimental distinction," Phys. Rev. Lett. 69, 3631 (1993).

9. V.G. Lyssenko, J. Erland, I. Balslev; K.-H. Pantke, B.S. Razbirin, and J.M. Hvam, "Nature of nonlinear four-wave mixing beats in semiconductors," Phys. Rev. B 48, 5720 (1993).

10. T. Yajima and Y. Taira, "Spatial optical parametric coupling of picosecond light pulses and transverse relaxation effects in resonant media," J. Phys. Soc. Japan 47, 1620 (1979).

11. J. Erland and I. Balslev, "Theory of quantum beat and polarization interference in four-wave mixing," Phys. Rev. B 48, 1765 (1994).

12. K.-H. Pantke and J.M. Hvam, "Nonlinear quantum beat spectroscopy in semiconductors," Int. J. Modern Phys. B 8, 73 (1994).

13. J. Erland, K.-H. Pantke, V. Mizeikis, V.G. Lyssenko, and J.M. Hvam, "Spectrally resolved four-wave mixing in semiconductors - influence of inhomogeneous broadening," to be published.

14. L.Q. Lampert, A. Compaan, and I.D. Abella, "Effects of nearly degenerate states on photon echo behaviour," Phys. Rev. A 4, 2022 (1971).

15. K.-H. Pantke, D. Oberhauser, V. G. Lyssenko, J.M. Hvam, and G. Weimann, "Coherent generation and interference of excitons and biexcitons in GaAs/Al $\mathrm{Ga}_{1-x} \mathrm{As}$," Phys. Rev. B 47, 2413 (1993).

16. D. Oberhauser, K.-H. Pantke, J.M. Hvam, G. Weimann, and C. Klingshirn, "Exciton scattering in quantum wells at low temperatures," Phys. Rev. B 47, 6827 (1993).

17. J. Erland, B.S. Razbirin, V.G. Lyssenko, K.-H. Pantke, and J.M. Hvam, "Nonlinear quantum beat spectroscopy of bound biexcitons in II-VI semiconductors," J. Crystal Growth 138, 800 (1994).

18. J. Feldmann, T. Meier, G. von Plessen, M. Koch, E.O. Göbel, P. Thomas, G. Bacher, C. Hartmann, H. Schweizer, W. Schäfer, and H. Nickel, "Coherent dynamics of exciton wave packets," Phys. Rev. Lett. 70, 3027 (1993).

19. D.J. Lovering, R.T. Phillips, G.J. Denton, and G.W. Smith, "Resonant generation of biexcitons in a GaAs quantum well," Phys. Rev. Lett. 68, 1880 (1992).

20. K. Leo, M. Wegener, J. Shah, D.S. Chemla, E.O. Göbel, T.C. Damen, S. Schmitt-Rink, and W. Schäfer, "Effects of polarization interactions on time-resolved degenerate four-wave mixing," Phys. Rev. Lett. 65, 1340 (1990).

21. B.F. Feuerbacher, J. Kuhl, and K. Ploog, "Biexciton contribution to the degenerate four-wave-mixing signal from a GaAs/Al ${ }_{x} G_{l-x}$ As quantum well," Phys. Rev. B 43, 2439 (1991).

22. M. Koch, J. Feldmann, E.O. Göbel, P. Thomas, J. Shah, and K. Köhler, "Coupling of exciton transitions associated with different quantum-well islands," Phys. Rev. B 48, 11480 (1993).

23. M. Kohl, D. Heitmann, S. Tarucha, K. Leo, and K. Ploog, "Optical investigation of the exciton transfer between growth islands of different well widths in GaAs/Al $\mathrm{Ga}_{1 . x} \mathrm{As}$ quantum wells," Phys. rev. B 39, 7736 (1989). 